\title{
Image Acquisition Rate Control Based on Object State Information in Physical and Image Coordinates
}

\author{
Feng-Li Lian and Shih-Yuan Peng \\ Department of Electrical Engineering, National Taiwan University \\ Taipei, Taiwan
}

\section{Introduction}

Recently, three-dimensional (3-D) vision applications play an important role in industry and entertainment. Among these applications, interactivity and scene reality are the two key features affecting the 3-D vision performance. Depending on the nature of the applications, the interactivity with the users (or the players) can be regarded as an important need, for example, in the applications of 3-D games and 3-D virtual reality. On the other hand, 3-D vision scenes can be generated based on physical or virtual models. In order to improve the scene reality, a new trend in 3-D vision system is to generate the scene based on some physical or real-world models.

For the 3-D reconstruction, research topics mainly focus on the static cases and/ or the dynamic cases. For the static cases, the reconstructed scenes do not change with the observed scenes. For the dynamic cases, the reconstructed scenes change with the observed scenes. For both static and dynamic cases, the 3-D positioning of moving objects in the scene is the major component of obtaining the object states in the physical coordinate. A typical 3D positioning system is shown in Figure 1. Cameras shown in Figure 1 are used to capture the images of moving objects in the physical coordinate. The image acquisition rate of Camera $X$ is denoted as Rate $X$. After one camera captures the images using its designated acquisition rate, the captured images are stored and analyzed in the computer. After analyzing the images, the object states, e.g., position and velocity, in the physical coordinate can be estimated by any 3-D positioning method.

The 3-D positioning plays an important role of obtaining the object states in the physical coordinate. In general, cameras are designated as the input devices for capturing images. Fundamental components of 3-D positioning are shown in Figure 2. First of all, images are captured by the input devices such as cameras. Before the image acquisition, the camera calibration should be done. Based on the calibration result, the relationship between the physical coordinate and the image coordinate in the captured images can be obtained. Since the quality of the captured images may be influenced by noise, some image processes should be performed to eliminate the noise. After the image processing, the captured images can be analyzed to extract the object information for further 3-D positioning. Finally, any 3$\mathrm{D}$ positioning method is used to find the object states such as the position and/ or velocity in the physical coordinate. 


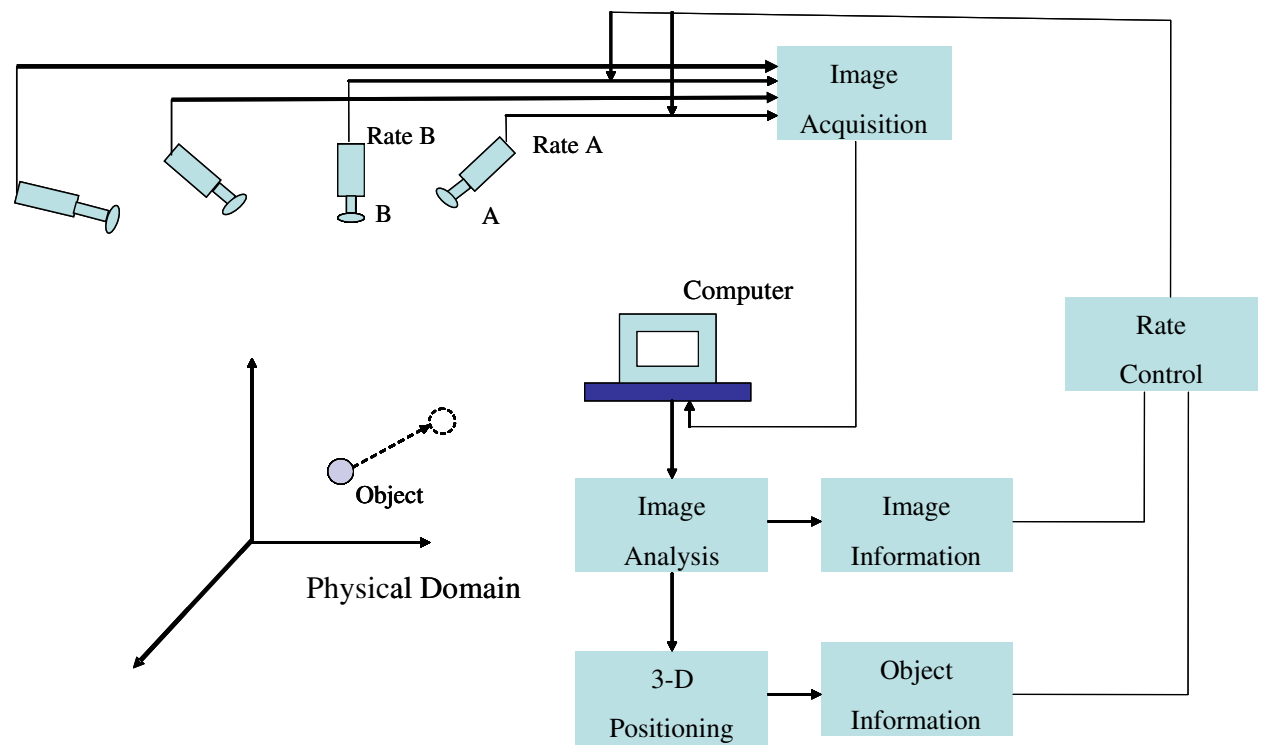

Fig. 1. A general 3-D positioning system

Due to the limitation on computational load, data storage, and communication bandwidth, the image acquisition rate may not be able to set at the highest rate for each camera. For example, the computation load of estimating the object trajectory in the physical coordinate may be too heavy and the data storage of all the captured images for future image analysis may be huge. Hence, a method of properly controlling the image acquisition rate is required for the purpose of saving the memory cost and/ or reducing the computational load. For designing a proper method to adaptively control the image acquisition rate, the object states in the image coordinate (after proper image processing) and/ or in the physical coordinate (after proper image/ positioning processing) should be considered in the acquisition rate control. Therefore, a rate control method as shown in Figure 2 is used to adjust the image acquisition rate based on the result of image analysis and the estimation of the object states.

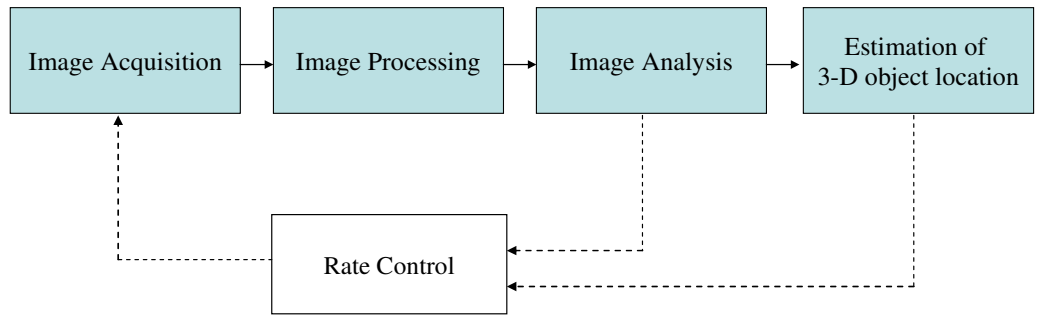

Fig. 2. Fundamental components of 3-D positioning

In this chapter, three methods of controlling the image acquisition rate are designed. With a suitable method for adjusting the acquisition rate, the ultimate goal is to reduce the computational load of the 3-D positioning, the memory cost, and the communication 
bandwidth for the captured images. The first method is to adjust the image acquisition rate by analyzing the object states in the physical coordinate. The second method considers the adjustment by analyzing the object states in the image coordinate. The third method deals with the issue of acquisition asynchronization among multiple cameras. The proposed methodologies have been experimentally tested using a two-camera setup within the MATLAB programming environment. Performance of using the proposed methods has been compared with that of using fixed-rate methods. Experimental results show that the proposed approaches achieve satisfactory performance and reduce computational load and data storage.

\subsection{Outline}

The rest of the chapter is organized as follows. Section 2 surveys related research work in camera control, 3-D positioning, object extraction, esimation of object position, database analysis, and visualization interface. Section 3 presents the three control methods of adjusting image acquistion rate based on image and physical coordinates. Section 4 illustrates experimental tests of the proposed image acqusition methods. Finally, Section 5 summarizes this chpater and discusses future tasks.

\section{Related research work}

In the literature, key 3-D vision applications for controlling the image acquisition rate is the 3D positioning of moving balls. Similar methodology can be applied to other objects or scenarios. A general framework of the 3-D positioning system is shown in Figure 3. The performance of image acquisition mainly depends on camera control. Different camera control methods can decide the quantity and quality of the information contained from the captured images for further image analysis. After the image acquisition, the captured images can be analyzed by the 3-D positioning methods for extracting the object states contained in these images. Once the image analysis of the object states is obtained, the analyzed result is stored in the database and further visualization interface is used to generate a 3-D animation. Related research works on these issues are summarized in detail in the following.

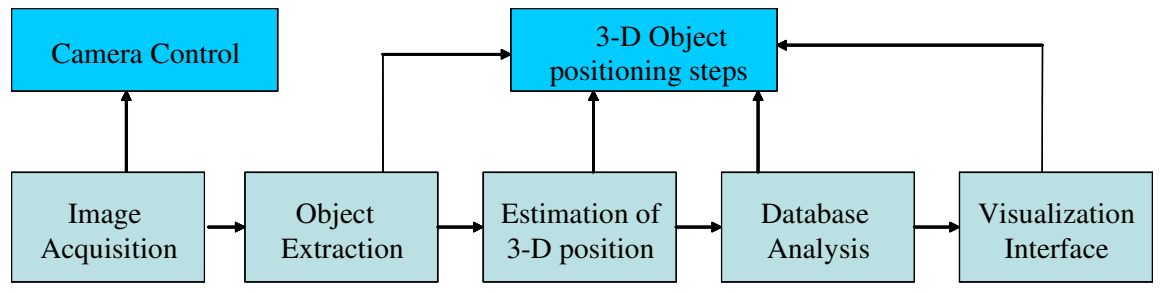

Fig. 3. General framework of 3-D image reconstruction system

\subsection{Camera control}

Images captured by cameras are used for further analysis in the 3-D reconstruction of scenes or the 3-D positioning of objects. Hence, the information obtained from the captured images is directly related to the issues on the location and the number to allocate these cameras. An example of the 3-D reconstruction from constrained views by using three cameras placed in the orthogonal directions is discussed in (Shin \& Shin, 1998). 
Generally speaking, the information in the captured images is influenced by the temporal and spatial factors. Hence, the information can be related to the image acquisition rate of cameras. If the image acquisition rate is high, more information can be obtained through the captured images. How ever, using a high acquisition rate takes much computational time for data processing and consumes a large amount of memory. Hence, properly controlling the image acquisition rate is a method that can reduce the computational load.

The amount of information in captured images is related to the image acquisition rate of the cameras. If the image acquisition rate is high, more information can be obtained through the captured images. However, a high acquisition rate might take more computation time for data processing and more storage memory. Therefore, the control method for adjusting image acquisition rate could help reduce the computational load as well as storage size. Since this kind of control methods adjust the sampling rate of input devices, they are classified as the handling of the temporal factors. In general, the inputs of the control methods for adjusting image acquisition rate are the current status of process system such as the computational load, or the states of interested object that are obtained by analyzing the captured images. The criterion for adjusting image acquisition rate might depend on the percentage of computational load and/ or the guaranteed amount of information in the captured images. For example, in (Hong, 2002), the criterion of the controlling the acquisition rate is specified as the amount of computational load and defined by the number of data management operations per unit time.

For the zoom and focus control of cameras, the main goal is to maintain required information of the captured images in the spatial resolution. The input for the zoom and focus control method is often regarded as the percentage of the target shown in the image search region. For example, in (Shah \& Morrell, 2004), the camera zoom is adjusted by the target position shown in the captured images. An adaptive zoom algorithm for tracking target is used to guarantee that a given percentage of particles can fall onto the camera image plane.

On the other hand, the pan-tilt motion controls of camera deal with the issue of guaranteeing enough spatial and temporal resolutions in the captured images. The camera is commanded to track the target and the target is required to be appeared in a specific region of the captured images. The inputs for the pan-tilt motion controls are often regarded as the percentage of target appeared in the search region and the velocity of camera motion to track the target in order to guarantee real-time performance. In (Wang et al., 2004), a realtime pan-tilt visual tracking system is designed to control the camera motion where the target is shown in the center area of the captured images.

\subsection{3-D ball extraction methods}

Commonly used methods to extract moving balls in the captured images can be classified based on the color information, geometric features of the balls, and the frame differencing technique. A comparison of related ball extraction methods is summarized in Table 1.

\begin{tabular}{|l|l|}
\hline Ball Extraction Method & References \\
\hline Color information & (Theobalt et al., 2004; Andrade et al., 2005; Ren et al., 2004) \\
\hline Geometric features & (Yu et al., 2004; Yu et al., 2003b; D'Orazio et al., 2002) \\
\hline Frame differencing & (Pingali et al., 2001) \\
\hline
\end{tabular}

Table 1. Methods of extracting ball movement from captured images 
First of all, a ball detection method based on the color of balls is studied in (Theobalt et al., 2004). The motion for a variety of baseball pitches is captured in an experimental environment where the floor and walls are covered with black carpet and cloth. Foreground object segmentation is facilitated by assigning the background with the same color. In order to record the rotation and spin of the ball along its trajectory, the entire surface of the ball is assigned with different markers. Similar idea used to track a ball by its color is also shown in (Andrade et al., 2005) and (Ren et al., 2004).

Secondly, the ball extraction can also be achieved by related geometric features of the ball. In (Yu et al., 2004) and (Yu et al., 2003b), several trajectory-based algorithms for ball tracking are proposed. From recorded image sequences, the candidates of possible ball trajectory are extracted. These trajectory candidates are detected by matching all the ball features such as circularity, size, and isolation. In (D'Orazio et al., 2002), a ball detection algorithm based on the circle hough transform of the geometric circularity is used for soccer image sequences.

Another method proposed in (Pingali et al., 2001) is based on the frame differencing between current and previous images. Supposing that the balls move fast enough and the background does not change, the balls in the foreground can be extracted by comparing the image sequences.

\subsection{Estimation of 3-D ball position}

Based on the extraction results, the ball states such as position and velocity can be estimated. Typical 3-D ball positioning methods include triangulation, trajectory fitting by physical models, and the estimation of the ball position by the Kalman filter. Related methods for the ball positioning are summarized in Table 2 .

\begin{tabular}{|l|l|}
\hline Methods for Ball Positioning & References \\
\hline Triangulation & (Ren et al., 2004) \\
\hline Trajectory fitting to physical models & (Ohno et al., 2000) \\
\hline Kalman filter & (Yu et al., 2003a) \\
\hline
\end{tabular}

Table 2. A comparison of different estimation methods for ball positioning

In (Ren et al., 2004), the 3-D positioning of a soccer is achieved by intersecting a set of two rays through two cameras and the observed ball projection on the ground in the images. Since these two rays usually have no intersection, a 3-D position of the object is employed as the point that has a minimum distance to both of these two rays.

In (Ohno et al., 2000), the ball position in a soccer game is estimated by fitting a mathematical ball model in the physical coordinate. The estimated position in each image frame depends on the initial position and the initial velocity of the ball in the physical coordinate. The overall trajectory of the soccer can then be determined by the equation specified by the gravity, air friction and timing parameters.

In (Yu et al., 2003a), the ball positions in the 3-D space are predicted by the Kalman filter which utilizes the information of past measured positions. By iteratively estimating the ball positions, the whole ball trajectory can be generated.

\subsection{Database analysis}

Database analysis focuses on calculating ball positions in all captured images or recorded video. Once all the ball positions are estimated, further analysis on the changes of ball trajectory can be obtained. For example, from the ball positions in the analyzed images or the ball trajectories in the physical coordinate, the speed and orientation can be calculated. 
In (Shum \& Komura, 2004), the speed and orientation of a baseball between two image frames are analyzed. The key events in a match can then be observed by reconstructing the baseball trajectory from the database. In (Yu et al., 2003b), the curves of soccer velocity in a match are generated for the analysis of special events. When a person touches the soccer, the trajectory of the soccer generates a local minimum point that changes with time. The local minimal velocity points in a velocity curve may also be caused by the ball bouncing that changes the soccer direction. The information of these events can help analyze the game strategy.

\subsection{Visualization interface}

The visualization interface is designed for users to watch the reconstructed animation such as the ball trajectory or the landing positions of a ball. In (Pingali et al., 2000), the authors show a virtual replay of a tennis game and the landing position. In (Pingali et al., 2001), a virtual replay environment which can let the users watch a replay at any speed and from any viewpoint is designed. Since the database stores all the ball information, the users can decide to visualize any interesting part of the game independently. If a visualization interface can also take into account of the practical situation in a match, the reality of the visualization can be improved. For example, an enrichment system of visualization interface is proposed in (Yan et al., 2004). This system can reconstruct a sport game in 3-D display and enrich the performance with related music and illustrations. Hence, the users can enjoy a comfortable viewing experience through a better visualization interface.

In this chapter, the methods for controlling image acquisition rate are designed. In order to test the control methods, several case studies of the 3-D object positioning are proposed. The ball extraction methods are based on the color information of the objects. Also, the triangulation method is used to estimate the position of balls in the physical coordinate. The visualization interface for the virtual replay of object positions is performed in the MATLAB programming environment.

\section{Methods for controlling image acquisition rate}

By selecting an appropriate image acquisition rate, the computational load of the 3-D positioning as well as the memory cost can be reduced. The proposed methods are based on the states of moving objects by analyzing the sequence of images obtained from the cameras. Two categories of information are used for designing the rate control methods. The first one is based on the object states in the physical coordinate and the second one is based on those directly in the image coordinate of captured images. Since multiple cameras are used and may have different acquisition rates, the captured images may not be synchronized. Hence, two types of reconstruction mechanisms are used for the information obtained in the image coordinate. Furthermore, a criterion is proposed to judge the performance of different image acquisition rates

\subsection{Control method based on object states in the physical coordinate (case A)}

After the 3-D positioning, the object states such as position and velocity in the physical coordinate can be computed. These object states can then be used as the key information for deciding the image acquisition rate. For example, when an object moves fast, the image 
acquisition rate should be set as high as possible to capture the object motion clearly. If an object moves slowly or is only in a stationary status, a high acquisition rate is not required. Using a high acquisition rate may waste the memory storage and the computational cost in image processing. On the other hand, using a low acquisition rate may lose the reality of the object motion. Hence, in order to balance the tradeoff between the computational cost and the accuracy of the 3-D positioning result, the control method for adjusting different image acquisition rates should be properly designed.

The proposed procedure is discussed in detail in the following. First of all, images are captured by multiple cameras. Since the quality of captured images may be influenced by noise, a spatial Gaussian filter is then used to smooth the captured images within the RGB color channels. Based on a set of predefined features of the objects in terms of the RGB color channels, the moving objects can be extracted from the images. After the objects are extracted, the region occupied by the objects in a captured image is stored in a new grayscale image. These gray-scale images can be further transformed into binary images by a specific threshold value. Some lighting influences on the objects may change the result of color analysis on the object surfaces. The object surfaces in the binary image might not be necessarily shown in a regular shape. Hence, the image dilation method can be used to enlarge the analyzed image area, and to improve the object shapes in these binary images. Finally, by the triangulation approach on the images, a set of 3-D information about the objects in the physical coordinate can be computed. Also, since the time of captured images and the corresponding estimated object position are recorded, the object velocity in the physical domain can be estimated. By analyzing the position and velocity of the object, the image acquisition rate for the next frame can then be adjusted based on the computed information.

In this case (named as Case A), the key feature of controlling the image acquisition rate is to analyze the object states in the physical coordinate. Assume that there are $\mathrm{N}$ levels of image acquisition rates, and each level only represents a specific range of values for the object states. Hence, by analyzing these object states, a corresponding image acquisition rate can be determined. Figure 4 shows an example of the mapping between the ball velocity (i.e., the object state) and the set of image acquisition rates. $\mathrm{V}_{0}, \mathrm{~V}_{1}, \mathrm{~V}_{2}, \ldots, \mathrm{V}_{\mathrm{N}}$, are the values of ball velocity in the physical coordinate. Rate 1 , Rate $2, \ldots$, Rate $\mathrm{N}$, are different levels of image acquisition rates arranged from the lowest value to the highest value. By analyzing the ball velocity, the corresponding image acquisition rate can be determined. That is, if the ball velocity is between $V_{i-1}$ and $V_{i}$, the corresponding image acquisition rate is set as Rate $i$.

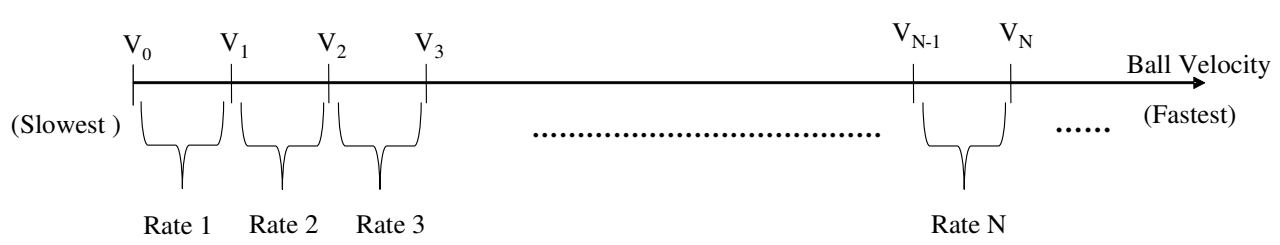

Fig. 4. An example of mapping different image acquisition rates by the ball velocity (object state) 
The pseudo code of the control algorithm for an example of three levels of acquisition rates is shown in Figure 5, where related variables used are defined in Table 3. The cases with more levels of image acquisition rates can be easily extended. Also, all the cameras are designed to adjust their image acquisition rates at the same time. For generating a complete replay, the object positions in the physical coordinate that are not calculated in the 3-D positioning can be predicted by using methods such as interpolation, extrapolation, or data fitting.

\section{$\underline{\text { Initialization }}$}

(All cameras are set to the high level of image acquisition rate at first.)

for each camera $C_{K} \quad(K=1 \sim n)$

$$
F_{C_{K}} \leftarrow F_{H}
$$

\section{Adjusting of image acquisition rate}

(All cameras adjust their image acquisition rate synchronously.)

$$
\text { for each incoming } V\left(T_{i}\right)
$$

if $\left(\left(V\left(T_{i}\right) !=\right.\right.$ empty $)$ and $\left(V\left(T_{i-1}\right) !=\right.$ empty $\left.)\right)$ then

Get $V\left(T_{i}\right)$ and $V\left(T_{i-1}\right)$ from database as bases

Generate $V_{P}\left(T_{i}+T_{L}\right)$ and $V_{P}\left(T_{i}+T_{N}\right)$

end if

$$
\begin{aligned}
& \text { for each camera } C_{K}(K=1 \sim n) \\
& \text { if }\left(\left(V_{S}<V_{P}\left(T_{i}+T_{L}\right)<V_{F}\right) \text { and }\left(V_{S}<V_{P}\left(T_{i}+T_{N}\right)<V_{F}\right)\right) \text { then } \\
& \qquad F_{C_{K}} \leftarrow F_{N}
\end{aligned}
$$

$$
\begin{gathered}
\text { else if }\left(\left(V_{P}\left(T_{i}+T_{L}\right)<V_{S}\right) \text { and }\left(V_{P}\left(T_{i}+T_{N}\right)<V_{S}\right)\right) \text { then } \\
F_{C_{K}} \leftarrow F_{L}
\end{gathered}
$$

else

$$
F_{C_{K}} \leftarrow F_{H}
$$

end if

\section{Fig. 5. Pseudo code of the algorithm in Case A}

When the objects are far aw ay from the cameras and move in a similar direction, the objects shown in the corresponding image coordinate are almost stationary. That is, the objects may move fast in the physical coordinate, but the information shown in the corresponding image coordinate does not reveal the fact. Therefore, the 3-D positioning result may not reveal correct object states in the physical coordinate. The type of failure is due to that little image information is used for the 3-D positioning. Hence, the control method should be modified to reflect the richness of image information from each individual camera. The amount of image information should be regarded as a decision factor for controlling the acquisition rate. The modified control methods are discussed in the following sections. 


\begin{tabular}{|c|c|c|}
\hline Symbol & Definition & Unit \\
\hline$C_{K}$ & index of camera $(K=1 \sim n)$ & none \\
\hline$F_{C_{K}}$ & the image acquisition rate for the specific index of camera & times/ sec \\
\hline $\max \left(F_{C_{K}}\right)$ & $\begin{array}{l}\text { the maximum image acquisition rate of the all cameras } \\
\qquad C_{K}(K=1 \sim n)\end{array}$ & times/ sec \\
\hline$F_{L}$ & the specific low level of image acquisition rate & times/ sec \\
\hline$F_{N}$ & the specific normal level of image acquisition rate & times/ sec \\
\hline$F_{H}$ & the specific high level of image acquisition rate & times/ sec \\
\hline$T_{i}$ & the recorded time of the captured image which is index $i$ & sec \\
\hline$T_{L}$ & $\begin{array}{l}\text { the corresponding image acquisition time to the lower level of } \\
\text { image acquisition rate }\left(T_{L}=1 / F_{L}\right)\end{array}$ & sec \\
\hline$T_{N}$ & $\begin{array}{l}\text { the corresponding image acquisition time to the normal level of } \\
\text { image acquisition rate }{ }_{\left(T_{N}=1 / F_{N}\right)}\end{array}$ & sec \\
\hline$P(t)$ & the object velocity in the pixel coordinate domain at time $t$ & $\mathrm{pixel} / \mathrm{sec}$ \\
\hline$P_{P_{C_{K}}}(t)$ & $\begin{array}{l}\text { the predictive object velocity in the image domain at time } t \text { (the } \\
\text { subscript } C_{K} \text { means the index of camera) }\end{array}$ & $\mathrm{pixel} / \mathrm{sec}$ \\
\hline$P_{S}$ & the threshold value of slow object velocity in the image domain & pixel/ sec \\
\hline$P_{F}$ & the threshold value of fast object velocity in the image domain & $\mathrm{pixel} / \mathrm{sec}$ \\
\hline$V(t)$ & the object velocity in the physical coordinate at time $t$ & $\mathrm{~mm} / \mathrm{sec}$ \\
\hline$V_{P}(t)$ & $\begin{array}{l}\text { the predictive object velocity in the physical coordinate at time } \\
\qquad t\end{array}$ & $\mathrm{~mm} / \mathrm{sec}$ \\
\hline$V_{S}$ & $\begin{array}{l}\text { the threshold value of slow object velocity in the physical } \\
\text { coordinate }\end{array}$ & $\mathrm{mm} / \mathrm{sec}$ \\
\hline$V_{F}$ & $\begin{array}{l}\text { the threshold value of fast object velocity in the physical } \\
\text { coordinate }\end{array}$ & $\mathrm{mm} / \mathrm{sec}$ \\
\hline
\end{tabular}

Table 3. The meanings of the symbols in the control algorithm

\subsection{Control method based on object states in the image coordinate (case $B$ and case $C$ )}

In Case A, the method for controlling the image acquisition rate is based on the object states in the physical coordinate. In this section, novel methods to control the image acquisition rate directly based on the object states in the image coordinate are proposed. The reason of using the object states in the image coordinate is that the acquisition rates can be effectively adjusted based on the actual available information instead of the projected information in the physical coordinate. Based on different synchronization scenarios among the cameras, two cases for adjusting the image acquisition rates are discussed. In the first case (named as Case B), each camera adjusts the image acquisition rate independently to other cameras, while in the second case (named as Case $\mathrm{C}$ ) all the cameras adjust their image acquisition rates cooperatively. 


\subsubsection{Independently controlling image acquisition rate (case $B$ )}

In Case B, each camera is designed to control its image acquisition rate independently based on the information in the image coordinate. The object states are first analyzed independently in the image coordinate of each camera and then each camera adjusts the acquisition rate based on the significance of the analyzed image. The significance can be used as a function of the mobility or the geometric feature of the object. Since all the cameras do not capture image simultaneously, the numbers of captured images at each camera are neither the same nor synchronized. Hence, during the 3-D reconstruction, the images from some cameras may be missed. To overcome this drawback, an interpolation or extrapolation method should be applied for those missing images. The advantage of only capturing the most significant images is to reduce the computational load for reconstructing useless images in the physical coordinate, and/ or the transmission bandwidth. The algorithm for the adjustment of three-level image acquisition rates is shown in Figure 6. The definition of the variables in the algorithm is also listed in Table 3. Cases with more levels of image acquisition rates can be easily extended.

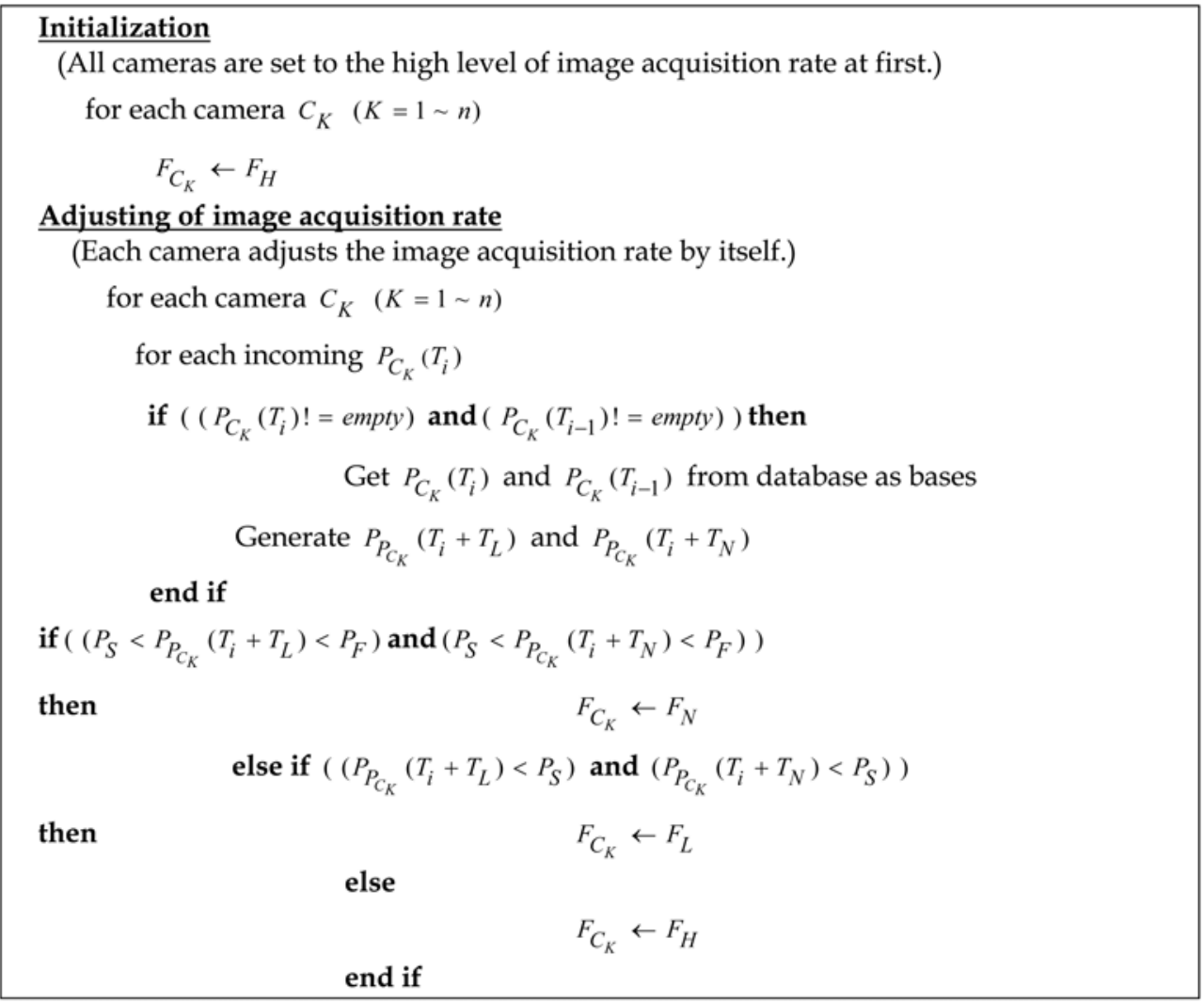

Fig. 6. Pseudo code of the algorithm in Case B

In Case A, all the cameras adjust their image acquisition rates simultaneously, so that the captured images are always in pairs for further processing. Hence, the object states in the 
physical coordinate can be directly reconstructed. In Case B, since these cameras adjust their image acquisition rate independently, the acquisition efficiency of each camera could be improved, but the captured images may not be necessarily in pairs. The missing images can be recovered by an interpolation or extrapolation estimation based on the captured images. Therefore, the 3-D reconstruction of the objects in the physical coordinate can also be performed.

However, in some scenarios, if the reconstruction by interpolation or extrapolation generates unacceptable errors, it is advised to increase the acquisition rates of some cameras in a cooperative way. The cooperation increases the acquisition rates of some cameras and hence improves the reconstruction performance. However, the total number of captured images is still smaller than that in Case A. Therefore, the computational cost is still reduced. The cooperation mechanism is discussed in next section.

\subsubsection{Cooperatively controlling image acquisition rates (case $\mathbf{C}$ )}

In this case, the method for controlling image acquisition rate is also based on the information obtained in the image coordinate. In additions, all cameras try to adjust its image acquisition rate synchronously. In order to achieve the synchronization, these acquisition rates should first be compared. The comparison works as follows. Each camera first predicts an image acquisition rate for the next image frame. The control algorithm then compares the prediction of each camera and then a designated image acquisition rate is chosen as the highest of all the predicted image acquisition rates. Finally, all the cameras adjust to their acquisition rates synchronously. Therefore, the captured images from all the cameras can be used for the 3-D reconstructions simultaneously.

The algorithm for the case of three levels of image acquisition rates is shown in Figure 7. The definition of the variables in the algorithm is also listed in Table 3. Cases with more levels of image acquisition rates can be easily extended. Initially, all the cameras are set to the higher level of image acquisition rate and, hence, capture some images in the meantime. Therefore, the set of captured images are in pairs. After that, the image is further analyzed and, because the image information obtained is different among these cameras, each camera may adjust its acquisition rate independently. Next, at each camera, the image acquisition rate for the next image frame is analyzed and predicted based on the captured images. In order to capture the next image frame synchronously, all the cameras are adjusted to the highest level of all the predicted image acquisition rates and the image frames can be guaranteed to be captured at the same time.

By using this algorithm, the cameras can compare their predicted image acquisition rates and adopt the highest rate for the next image frame. Since the algorithm in Case $\mathrm{C}$ adjusts the image acquisition rate before analyzing the object information in the physical coordinate, this algorithm could performs more efficiently than that of Case A. A preliminary comparison of the three control algorithms is listed in Table 4.

In addition to the synchronization effect and reconstruction performance, other factors can also be considered when selecting a suitable algorithm for controlling the image acquisition rate. The most important concept is that the adjustment of the acquisition rate should depend on the image quality captured by each camera. In order to characterize the outcome of the proposed control methods, a performance criterion is proposed in the next section. 


\section{$\underline{\text { Initialization }}$}

(All cameras are set to the high level of image acquisition rate at first.)

for each camera $C_{K}(K=1 \sim n)$

$$
F_{C_{K}} \leftarrow F_{H}
$$

\section{Adjusting of image acquisition rate}

(All cameras adjust their image acquisition rate synchronously.)

for each camera $C_{K} \quad(K=1 \sim n)$

$$
\text { if }\left(\left(P_{C_{K}}\left(T_{i}\right) !=\text { empty }\right) \text { and }\left(P_{C_{K}}\left(T_{i-1}\right) !=\text { empty }\right)\right) \text { then }
$$

Get $P_{C_{K}}\left(T_{i}\right)$ and $P_{C_{K}}\left(T_{i-1}\right)$ from database as bases

end if

$$
\text { Generate } P_{P_{C_{K}}}\left(T_{i}+T_{L}\right) \text { and } P_{P_{C_{K}}}\left(T_{i}+T_{N}\right)
$$

$$
\begin{aligned}
& \text { for each incoming } P_{C_{K}}\left(T_{i}\right) \\
& \begin{array}{c}
\text { if }\left(\left(P_{S}<P_{P_{C_{K}}}\left(T_{i}+T_{L}\right)<P_{F}\right) \text { and }\left(P_{S}<P_{P_{C_{K}}}\left(T_{i}+T_{N}\right)<P_{F}\right)\right) \text { then } \\
F_{C_{K}} \leftarrow F_{N}
\end{array} \\
& \text { else if }\left(\left(P_{P_{C_{K}}}\left(T_{i}+T_{L}\right)<P_{S}\right) \text { and }\left(P_{P_{C_{K}}}\left(T_{i}+T_{N}\right)<P_{S}\right)\right) \text { then }
\end{aligned}
$$$$
F_{C_{K}} \leftarrow F_{L}
$$$$
\text { else }
$$$$
\text { end if }
$$

$$
F_{C_{K}} \leftarrow F_{H}
$$

Find $\max \left(F_{C_{K}}\right)$

Set the image acquisition rate of all cameras as $\max \left(F_{C_{K}}\right)$

Fig. 7. Pseudo code of the algorithm in Case C

\begin{tabular}{|c|c|c|c|}
\hline & Case A & Case B & Case C \\
\hline The time to carry out the algorithm & Long & Short & Normal \\
\hline Are captured images always in pairs? & Yes & No & Yes \\
\hline
\end{tabular}

Table 4. The comparison of control algorithms designed in this study

\subsection{Criterion for characterizing image acquisition}

These methods proposed for controlling the image acquisition rate could reduce computational cost and memory space for the captured images. For the 3-D positioning, the object position, for example, in the physical coordinate can be estimated based on the information of analyzing the captured images. In order to compare the performance of different control methods, the following criterion is designed:

$$
R=W_{s} \times s-W_{d} \times d
$$


where $s$ denotes the saving percentage of image quantity, $d$ denotes a reference value representing the percentage of image distortion, and $W_{s}, W_{d}$ are two weighting factors. Specifically, $s$ can be described as follows:

$$
s=1-\frac{N_{1}}{N_{2}},
$$

where $N_{1}$ denotes the number of captured images when carrying out the control method and $N_{2}$ denotes the number of captured images when all the cameras are set at the highest acquisition rate. For example, if 240 images are captured using the highest acquisition rate, and only 200 images are recorded by performing one of control methods, then the percentage of image saving is estimated as follows:

$$
s=1-\frac{200}{240} \cong 0.167=16.7 \% .
$$

Furthermore, $W_{s}$ can be regarded as a function of memory saving. For example, the function can be defined as follows:

$$
W_{s}=\alpha(\xi),
$$

where $\xi=M_{1} / M_{2}$ and $M_{1}$ denotes the quantity of memory used to record all the captured images by using the highest acquisition rate, and $M_{2}$ denotes the quantity of memory assigned by the computer in advance. For example, if it needs $100 \mathrm{MB}$ to record all the captured images and the memory size assigned by the computer is $80 \mathrm{MB}, \xi$ can be calculated as follows:

$$
\xi=\frac{100}{80}=1.25
$$

If $\xi>1$, then saving the number of captured images can be considered an important goal. $W_{d}$ can be regarded as a function of the object states and the image acquisition rate. For example, $W_{d}$ is described as follows:

$$
W_{d}=\beta(v, k),
$$

where $v$ characterizes the object states such as position or velocity, and $k$ is the highest acquisition rate used in a practical application. If the object states have a large impact on the image processing (for example, the object speed is large), and the image acquisition rate is low, it is likely that the outcome of the image processing will be distorted and the value of $W_{d}$ should be increased. In summary, $W_{s} \times s$ can be regarded as the advantage for carrying out the control method, while $W_{d} \times d$ can be regarded as the disadvantage for carrying out the control method. 
When the value of $R$ is equal to zero, the performance of the control method is just the same as that without any acquisition rate control because all the images are just captured by the highest image acquisition rate and occupy all the available memory. Hence, in order to guarantee the performance of the control method, the value of $R$ should be a positive number. If several control methods are available for the users to choose, they can be compared based on the criterion.

\section{Experimental results}

In this section, the 3-D positioning of solid balls are experimentally tested for the three methods of controlling image acquisition rates. The test-bed is shown in Figure 8(a). The image coordinates of the two cameras is shown in Figure 8(b), where the camera centers are denoted as $\mathrm{P}_{1}$ and $\mathrm{P}_{2}$, respectively, and $\pi_{1}$ and $\pi_{2}$ are the image frames of the two camera, repsectively. Since three balls of different colors are used in these experiments, the foreground object segmentation can be easily performed. The background of the scene is only white broads. Also, two cameras are used to capture the images which are stored and analyzed at one single computer.

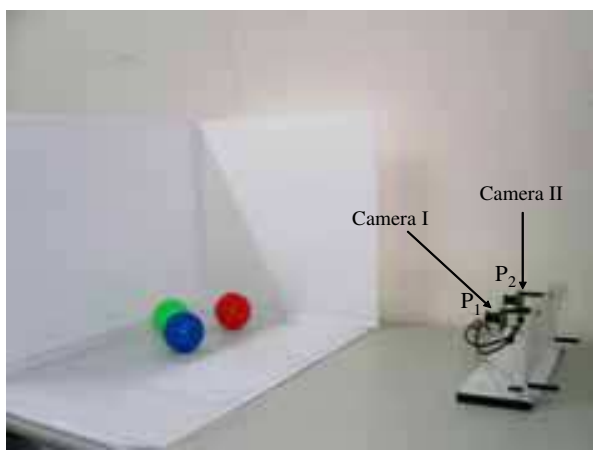

(a)

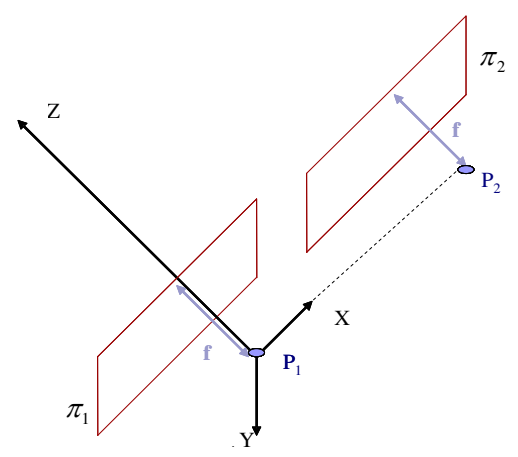

(b)

Fig. 8. (a) A snapshot of experimental environment for 3-D positioning, (b) the image coordinates of the two cameras

In this study, assume that the radius of these balls is known in advance. Hence, the goal of the 3-D positioning is only to identify the center of these balls. In order to locate the ball centers in the physical coordinate, the ball center shown in each camera should be processed first by proper image processing operations. Once the position of the ball center in the image coordinate of each camera is found, the position of ball center in the physical coordinate can then be computed by the triangulation method used in the 3-D mapping.

\subsection{Image processing results}

The captured images are stored in the memory of the computer and processed by the image processing step. First of all, the original captured images loaded from the memory are fullcolor images, i.e., with red, green and blue color channels. The spatial Gaussian filter is then used to process these color channels, independently. In order to distinguish these three balls shown in the image, each ball is identified from the image by the color analysis. Supposing 
that a ball region is extracted after the color analysis, the region is stored and transformed into a gray-scale image. The gray-scale image can be further transformed into a binary image. Finally, the image dilation method is used to further characterize the shape of ball region shown in the binary image.

In the color analysis step, all pixels in a captured image are processed by a color analysis method. That is, a pixel shown in the captured images can be judged as either a part of color ball or a part of background. When a pixel does not belong to the region of the three balls, the pixel is regarded as a part of background. This color analysis method is designed to process all pixels in the captured image. Supposing that the balls are not blocked by others, this color analysis method can identify every ball region. An example of using the color analysis method to find a ball region is shown in Figure 9.

In Figure 9(a), an original captured image is shown. Next, the extraction result of the green ball is shown in Figure 9(b), where a binary image is obtained. It is clear that the ball shape is incorrect after the color analysis. Hence, the image dilation method is used to further improve the result of ball region extraction. The result after the image dilation is shown in Figure 9(c), where the ball shape is better than that shown in Figure 9(b).

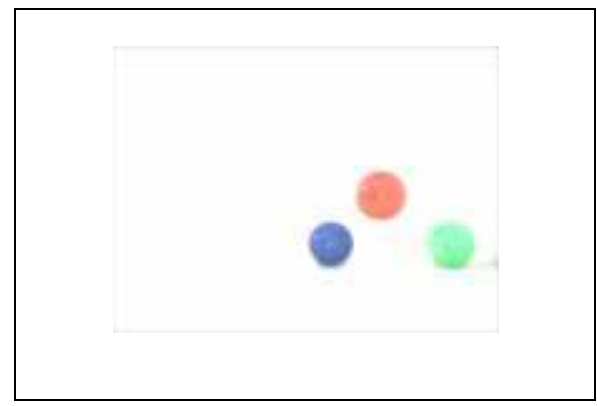

(a)

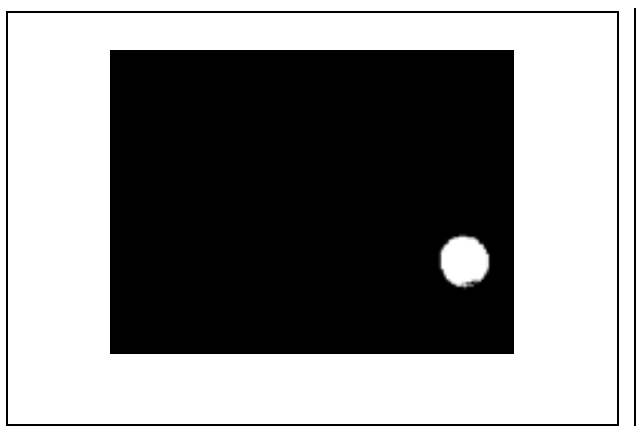

(b)

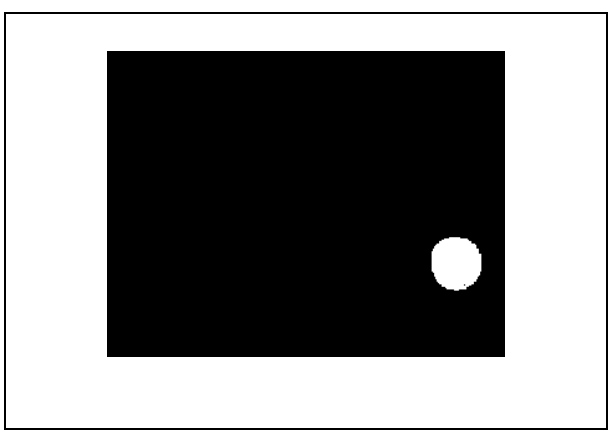

(c)

Fig. 9. The results of ball extraction and image dilation in the captured image. (a) The original image. (b) After ball extraction. (c) After image dilation

\subsection{3-D positioning results}

In this study, the target for 3-D positioning are the centers of the balls. In order to find the positions of ball centers in the physical domain, the ball centroid shown in images are first 
calculated by the image processing step discussed in previous section. Once the positions of ball centroid shown in images are found, the position of ball center in the physical domain can be found by the triangulation method. Supposing that a ball center $s$ is the target for 3D positioning, an example of the corresponding points of $s$ in the image planes of the two cameras used in this study is shown in Figure 10.

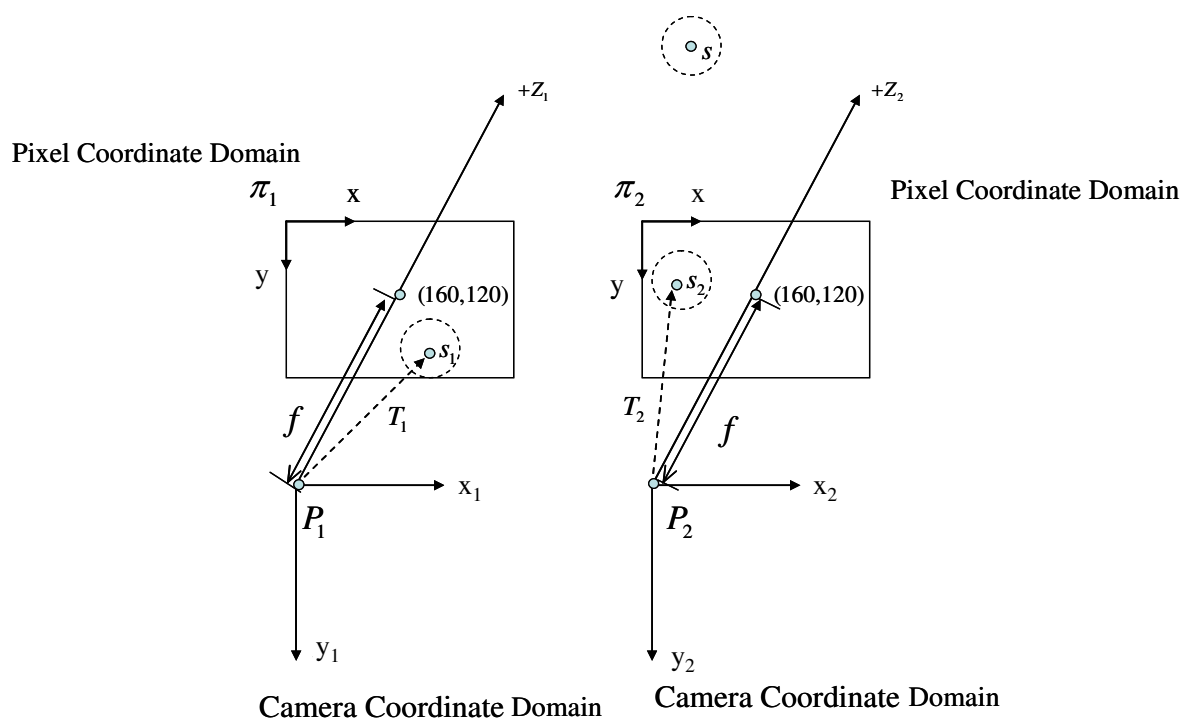

Fig. 10. An example for describing the corresponding points of a ball center in the image planes

The centers of the two camera coordinate domains are denoted by $P_{1}$ and $P_{2}$, respectively, and the image planes are denoted by $\pi_{1}$ and $\pi_{2}$, respectively. Assume that the range of the image plane is $320 * 240$ in pixel and the center of the image coordinate is then denoted as $(160,120)$. The focal length is denoted by $f$. The ball center $s$ is the target of the 3-D positioning, and the corresponding point of $s$ shown in the two image coordinates are denated as $s_{1}$ and $s_{2}$, respectively. Hence, the coordinates of $s_{1}$ and $s_{2}$ in $\pi_{1}$ and $\pi_{2}$, respevtively, can be described as follows:

$$
\begin{aligned}
& s_{1}=\left(x_{1}, y_{1}\right) \\
& s_{2}=\left(x_{2}, y_{2}\right)
\end{aligned}
$$

Supposing that the unit of the focal length $f$ is in terms of pixel, the two 3 -D vectors $T_{1}$ and $T_{2}$ can be described as follows

$$
\begin{aligned}
& T_{1}=\left(x_{1}-160, y_{1}-120, f\right) \\
& T_{2}=\left(x_{2}-160, y_{2}-120, f\right)
\end{aligned}
$$


where $T_{1}$ denotes the 3-D vector from $P_{1}$ to $s_{1}$ and $T_{2}$ denotes the 3 -D vector from $P_{2}$ to $s_{2}$. After finding the two vectors, the position of $s$ can be calculated by the triangulation method as shown in Figure 11.

In Figure 11, the coordinate domain is in the physical domain. Line $L_{1}$ in the physical domain can be determined by the information of Point $P_{1}$ and Vector $T_{1}$. Line $L_{2}$ in the physical domain can be determined by the information of Point $P_{2}$ and Vector $T_{2}$. On Line $L_{1}, s_{1}$ ' is the point closest to Line $L_{2}$, and, on Line $L_{2}, s_{2}$ ' is the point closest to Line $L_{1}$. Point $s$ is the target point for 3-D positioning and is calculated by finding the midpoint of $s_{1}$, and $s_{2}$ '.

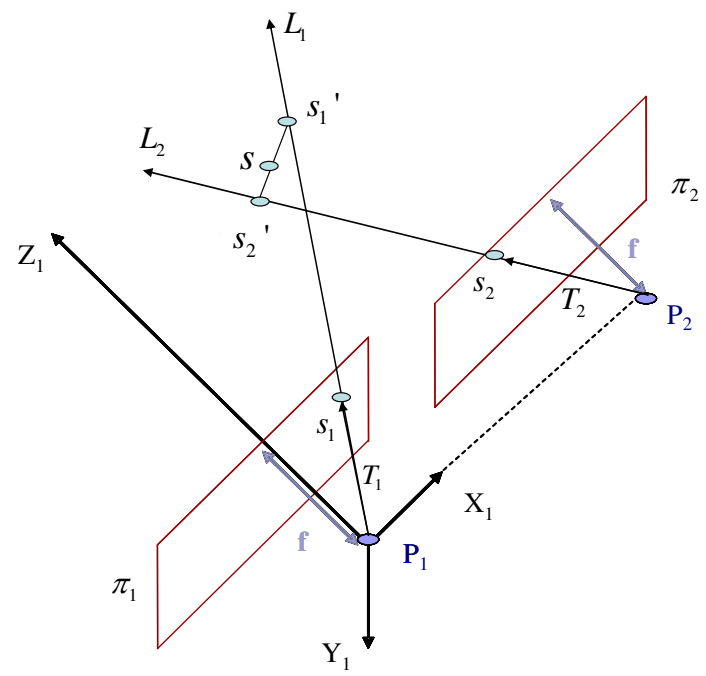

Fig. 11. An example of finding the position of ball center in the physical domain by the triangulation method

After all the captured images are processed by the 3-D positioning step, the trajectories of these balls can be reconstructed in a virtual environment, for example, in the 3-D plotting in MATLAB. In the virtual environment, these balls can be shown in different viewpoints. An example of the 3-D positioning for the three balls is shown in Figure 12. The original placement of the three balls is shown in Figure 12(a), and the 3-D positioning result in the virtual environment from three different view points are shown in Figure 12(b), (c), and (d), respectively.

For testing the three cases of rate control, three levels of image acquisition rates are used. They are characterized as the higher level: 4 frames/ sec, the normal level: 2 frames/ sec, and the lower level: 1 frames/ sec. In each case, twenty experiments are tested for 60 seconds. In each test, the ball velocity in the physical coordinate can be calculated based on the 3-D positioning of the ball center and the sampling time interval. Hence, two threshold values $V_{S}$ and $V_{F}$ used in Case A can then be determined based on the analyzed data. In the study, $V_{S}$ and $V_{F}$ are set as 32.47 and $48.46 \mathrm{~mm} / \mathrm{sec}$, respectively. Similarly, the threshold values $P_{S}$ and $P_{F}$ used in Case B and Case C are set as 9.80 and $17.05 \mathrm{pixel} / \mathrm{sec}$, respectively. 


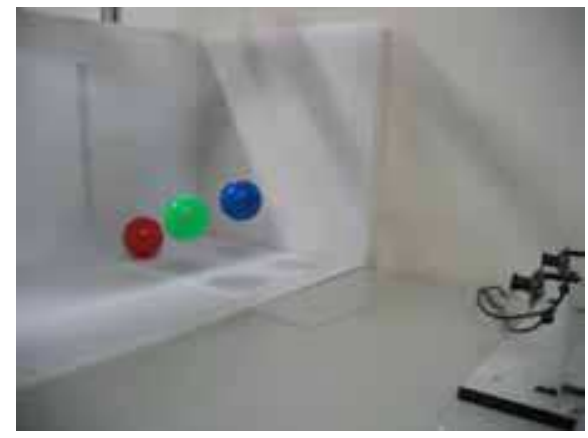

(a)

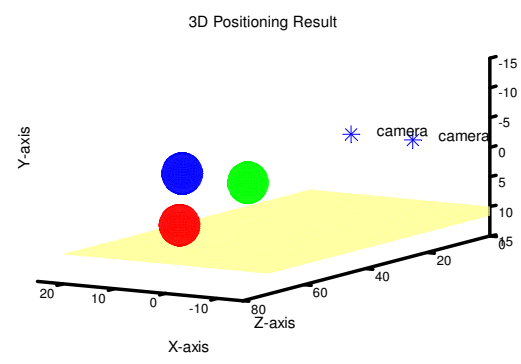

(c)

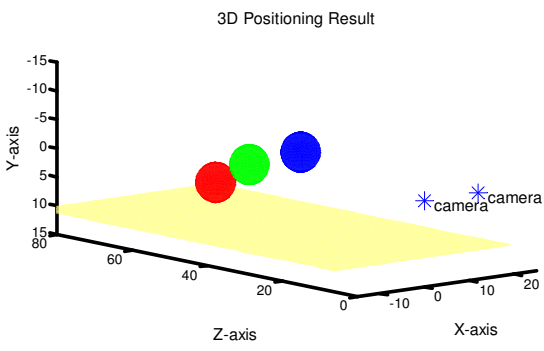

(b)

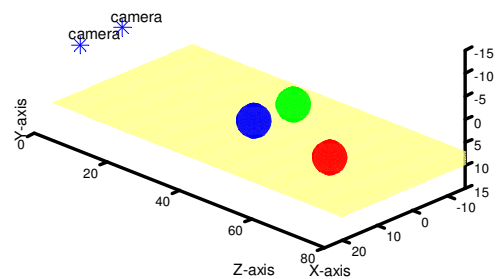

(d)

Fig. 12. An example of the 3-D positioning of the three balls. (a) A snapshot of the original placement. (b) The 3-D positioning reconstructed in the MATLAB environment. (c) and (d) The 3-D positioning results shown in the different viewpoints

Furthermore, for the evaluation of the control methods, the root-mean-square-error (RMSE) of the difference between the tests with and without rate control is calculated. The definition of RMSE is described as follows:

$$
R M S E=\sqrt{\frac{1}{n} \sum_{i=1}^{n}\left(q_{i}-\hat{q}_{i}\right)^{2}}
$$

where $n$ is the number of predicted results, $q$ denotes the original image quality, and $\hat{q}$ denotes the predicted image quality. When there are no corresponding images, the 3-D positioning results are estimated by the interpolation, extrapolation, or data fitting to a line by the least squares approximation.

In this study, the three control methods discussed in Section 3 are performed and compared by the average value of RMSE. In each case, five similar experiments are performed. The images in the five experiments are captured first by the higher level of image acquisition rate. Each experiment lasts for 60 seconds and 240 images are captured in each experiment. The result of the percentage of image saving is shown in Table 5. Since the original 3-D positioning results in the five experiments are calculated in advance, the values of RMSE for different control methods can be calculated. By using the interpolation method to predict 
unknown 3-D positions, the calculated results for RMSE is shown in Table 6. By using the extrapolation method to predict unknown 3-D positions, the calculated results for RMSE is shown in Table 7. Finllay, by using the method of least squares approximation to predict unknown 3-D positions, the calculated results for RMSE is shown in Table 8. Also, the timing data of the image acquisition by uisng the three control methods and the fixed rate cases are shown in Figure 13.

When all the images are captured by a fixed, normal image acquisition rate, the percentage of image saving is the smallest. In Case $\mathrm{C}$, the image acquisition rate is adjusted before comparing the image processing result of the two cameras. Once one camera determines the time to capture next image, the other camera also capture the image no matter what its original decision is. Hence, the percentage of image saving for Case $\mathrm{C}$ is smaller than that of Case A and Case B.

For the prediction performance, since Case $\mathrm{C}$ acquires more images than Case $\mathrm{A}$ and Case $\mathrm{B}$ do, the RMSE for Case C are smaller than those for Case A and Case B. In Case B, the two cameras do not utilize identical image acquisition rate synchronously. Hence, the position of the ball in the image coordinate is then predicted. In Case A and Case B, the image acquisition rate for the two cameras is adjusted synchronously. Therefore, the RMSE in Case $\mathrm{B}$ is larger than those Case A and Case C.

\begin{tabular}{|c|c|c|c|c|c|}
\hline & \multicolumn{5}{|c|}{ Percentage of image saving } \\
\hline $\begin{array}{c}\text { Index of } \\
\text { experiments }\end{array}$ & Case A & Case B & Case C & $\begin{array}{c}\text { Fixed rate } \\
\text { (normal level) }\end{array}$ & $\begin{array}{c}\text { Fixed rate } \\
\text { (low er level) }\end{array}$ \\
\hline 1 & $71.67 \%$ & $70.42 \%$ & $52.08 \%$ & $50 \%$ & $75 \%$ \\
\hline 2 & $71.67 \%$ & $70.21 \%$ & $54.58 \%$ & $50 \%$ & $75 \%$ \\
\hline 3 & $72.50 \%$ & $70.21 \%$ & $56.67 \%$ & $50 \%$ & $75 \%$ \\
\hline 4 & $72.92 \%$ & $70.63 \%$ & $63.75 \%$ & $50 \%$ & $75 \%$ \\
\hline 5 & $72.92 \%$ & $68.13 \%$ & $52.08 \%$ & $50 \%$ & $75 \%$ \\
\hline Average & $72.34 \%$ & $69.92 \%$ & $55.83 \%$ & $50 \%$ & $75 \%$ \\
\hline
\end{tabular}

Table 5. The percentage of image saving in the experiments

\begin{tabular}{|c|c|c|c|c|c|}
\hline & \multicolumn{5}{|c|}{ RMSE (by the interpolation method) (Unit: mm) } \\
\hline $\begin{array}{c}\text { Index of } \\
\text { experiments }\end{array}$ & Case A & Case B & Case C & $\begin{array}{c}\text { Fixed rate } \\
\text { (normal level) }\end{array}$ & $\begin{array}{c}\text { Fixed rate } \\
\text { (lower level) }\end{array}$ \\
\hline 1 & 32.122 & 63.279 & 25.957 & 22.951 & 33.863 \\
\hline 2 & 33.417 & 54.744 & 33.129 & 22.347 & 35.781 \\
\hline 3 & 29.328 & 47.472 & 26.531 & 20.588 & 30.771 \\
\hline 4 & 33.403 & 68.569 & 32.773 & 23.019 & 34.769 \\
\hline 5 & 31.169 & 109.168 & 27.352 & 21.224 & 32.368 \\
\hline Average & 31.888 & 68.646 & 29.142 & 22.026 & 33.510 \\
\hline
\end{tabular}

Table 6. The RMSE of prediction performance when all unknown 3-D positioning results are predicted by the interpolation method 


\begin{tabular}{|c|c|c|c|c|c|}
\hline & \multicolumn{5}{|c|}{ RMSE (by the extrapolation method) (Unit: mm) } \\
\hline $\begin{array}{c}\text { Index of } \\
\text { experiments }\end{array}$ & Case I & Case II & Case III & $\begin{array}{c}\text { Fixed rate } \\
\text { (normal level) }\end{array}$ & $\begin{array}{c}\text { Fixed rate } \\
\text { (lower level) }\end{array}$ \\
\hline 1 & 56.811 & 148.509 & 57.446 & 43.265 & 59.658 \\
\hline 2 & 67.293 & 103.541 & 63.782 & 44.111 & 63.331 \\
\hline 3 & 60.500 & 109.406 & 57.555 & 36.308 & 55.947 \\
\hline 4 & 59.374 & 134.716 & 67.257 & 41.649 & 55.583 \\
\hline 5 & 61.874 & 225.318 & 58.105 & 39.891 & 61.883 \\
\hline Average & 61.170 & 144.298 & 60.229 & 41.045 & 59.280 \\
\hline
\end{tabular}

Table 7. The RMSE of prediction performance when all unknown 3-D positioning results are predicted by the extrapolation method

\begin{tabular}{|c|c|c|c|c|c|}
\hline & \multicolumn{5}{|c|}{ RMSE (by the method of data fitting to a line) (Unit: mm) } \\
\hline $\begin{array}{c}\text { Index of } \\
\text { experiments }\end{array}$ & Case A & Case B & Case C & $\begin{array}{c}\text { Fixed rate } \\
\text { (normal level) }\end{array}$ & $\begin{array}{c}\text { Fixed rate } \\
\text { (lower level) }\end{array}$ \\
\hline 1 & 56.628 & 122.772 & 51.346 & 39.219 & 56.959 \\
\hline 2 & 62.361 & 103.874 & 63.106 & 41.516 & 59.385 \\
\hline 3 & 57.299 & 102.364 & 52.468 & 36.267 & 53.805 \\
\hline 4 & 59.218 & 136.043 & 59.807 & 38.435 & 54.829 \\
\hline 5 & 56.965 & 210.031 & 53.714 & 41.380 & 56.788 \\
\hline Average & 58.494 & 135.017 & 56.088 & 39.363 & 56.353 \\
\hline
\end{tabular}

Table 8. The RMSE of prediction performance when all unknown 3-D positioning results are predicted by the method of data fitting to a line

Finally, the performance criterion, defined in Section 3.3, of these three cases is summarized in Table 9. The performance criteria in Case A are larger than those in Case B and Case C. Hence, the performance of Case A is the best. Also, it is advised to use a control method with a positive $R$. Therefore, the performance of Case B is not good enough. Also, the statistic suggests that the performance of using interpolation to predict the missing data is better than that of using extrapolation and data fitting.

\begin{tabular}{|c|c|c|c|}
\hline & \multicolumn{3}{|c|}{$R$} \\
\cline { 2 - 4 } & Case A & Case B & Case C \\
\hline Interpolation & 0.2440 & -0.1614 & 0.1139 \\
\hline Extrapolation & -0.1842 & -1.0421 & -0.3477 \\
\hline Data fitting & -0.1520 & -0.9354 & -0.2723 \\
\hline
\end{tabular}

Table 9. The performance criterion $R$

In summary, Case A has a better result in the percentage of image saving and the performance criterion. For Case B, due to the asynchronization in image acquisition, the 3-D 
positioning results cannot be predicted properly and may generate bigger errors than that in Case A and Case C. Therefore, if the cameras can be placed in an appropriate position to avoid the problem of obtaining too little object information, Case A should be a better choice for practical application than Case C.

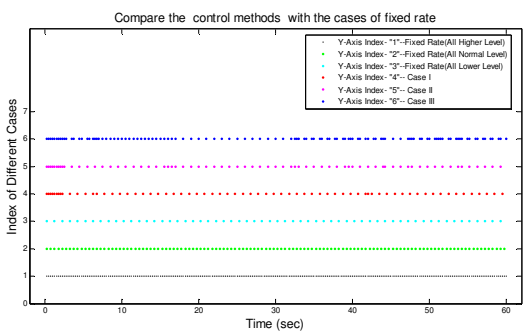

(a)

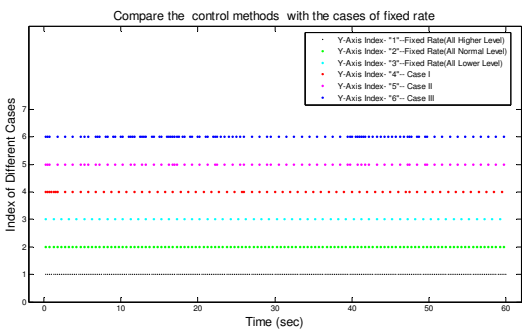

(c)

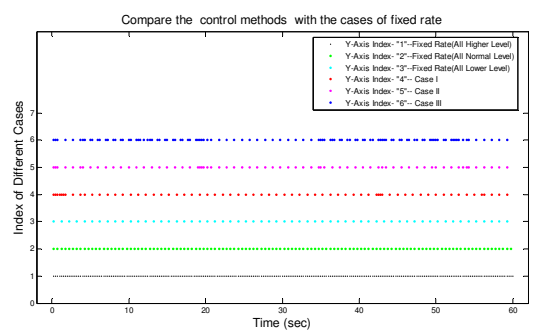

(b)

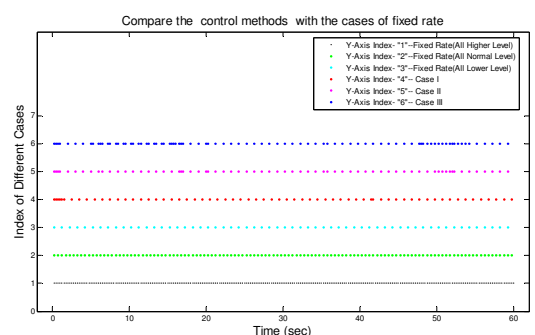

(d)

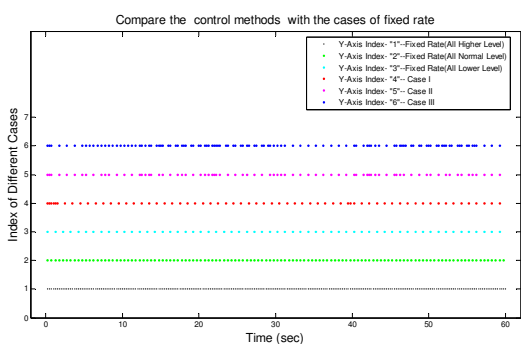

(e)

Fig. 13. The timing comparison for different image acquisition mechanisms by using the three control methods and the fixed rate cases. (a)-(e) The five experiments performed

\section{Conclusions and future work}

In this study, three methods for controlling image acquisition rate are designed and analyzed. In order to verify the control methods, the 3-D positioning of balls is used to compare the performance of the three control methods in terms of the percentage of saving images and the accuracy of the 3-D positioning results. Experimental results show that the 
calculating results for the percentage of saving images in Case $\mathrm{C}$ is smaller than that in Case $\mathrm{A}$ and Case B. However, since Case $\mathrm{C}$ adopts more images to predict the motion of the balls in the physical domain, the accuracy of the 3-D positioning result in Case $\mathrm{C}$ is better than that in Case A and Case B. The accuracy for the 3-D positioning result for Case B is worse than that in Case A and Case C. In order to compare the performance of the three cases, a performance criterion for judging these control methods is proposed. In practical applications, cameras should be placed or moved in an appropriate position to avoid the problem of getting too little object information from the images.

In the future, the following three tasks can be further performed. The first task is to use advanced mathematical methods for predicting the positions of the interested objects in the physical domain. The second task is to design more advanced control methods for better characterize the object states. The third task is to test the scenarios with more cameras and interested objects for testing the complexity achieved by the proposed control methods.

\section{Acknowledgement}

This work was supported in part by the National Science Council, Taiwan, ROC, under the grants: NSC 95-2221-E-002-303-MY3, and NSC 96-2218-E-002-030, and by DOIT/ TDPA: 96EC-17-A-04-S1-054.

\section{References}

Andrade, E.L.; Woods, J.C.; Khan, E. \& Ghanbrai M. (2005) Region-Based Analysis and Retrieval for Tracking of Semantic Objects and Provision of Augmented Information in Interactive Sport Scenes, IEEE Transactions on Multimedia, Vol. 7, No. 6, (Dec. 2005) pp. 1084-1096, ISSN: 1520-9210

D’Orazio, T.; Ancona, N.; Cicirelli, G. \& Nitti, M. (2002) A Ball Detection Algorithm for Real Soccer Image Sequences, Proceedings of the 16th International Conference on Pattern Recognition, pp. 210-213, ISBN: 0-7695-1685-x, Quebec, Canada, Aug. 2002, Institute of Electrical and Electronics Engineers, Piscataw ay

Hong, S.M. (2002) Steady-State Analysis of Computational Load in Correlation-Based Image Tracking, IEE Proceedings on Vision, Image, and Signal Processing, Vol. 149, No. 3, (June 2002) pp. 168-172, ISSN: 1350-245X

Iw ase, H. \& Murata A. (2001) Discussion on Skillful Baseball Pitch Using Three-Dimensional Cinematographic Analysis. Comparison of Baseball Pitch between Skilled and Unskilled Pitchers, Proceedings of the IEEE International Conference on Systems, Man, and Cybernetics, pp. 34-39, ISBN: 0-7803-7087-2, Tucson, AZ, USA, Oct. 2001, Institute of Electrical and Electronics Engineers, Piscataw ay

Ohno, Y.; Miura, J. \& Shirai Y. (2000) Tracking Players and Estimation of the 3D Position of a Ball in Soccer Games, Proceedings of the IEEE International Conference on Pattern Recognition, pp. 145-148, ISBN: 0-7695-0750-6, Barcelona, Spain, Sep. 2000, Institute of Electrical and Electronics Engineers, Piscataw ay

Pingali, G.; Opalach, A.; Jean, Y. \& Carlbom, I. (2001) Visualization of Sports Using Motion Trajectories: Providing Insights into Performance, Style, and Strategy, Proceedings of 
the IEEE International Conference on Visualization, pp. 75-82, ISBN: 0-7803-7200-x, San Diego, CA, USA, Oct. 2001, Institute of Electrical and Electronics Engineers, Piscataway

Pingali, G.; Opalach, A. \& Jean, Y. (2000) Ball Tracking and Virtual Replays for Innovative Tennis Broadcasts, Proceedings of the IEEE International Conference on Pattern Recognition, pp. 152-156, ISBN: 0-695-0750-6, Barcelona, Spain, Sep. 2000, Institute of Electrical and Electronics Engineers, Piscataw ay

Ren, J.; Orwell, J.; Jones, G.A. \& Xu, M. (2004) A General Framework for 3D Soccer Ball Estimation and Tracking, Proceedings of the IEEE International Conference on Image Processing, pp. 1935-1938, ISBN: 0-7803-8554-3, Genova, Italy, Oct. 2004, Institute of Electrical and Electronics Engineers, Piscataw ay

Shah, H. \& Morrell, D. (2004) An Adaptive Zoom Algorithm for Tracking Targets Using Pan-Tilt-Zoom Cameras, Proceedings of the IEEE International Conference on Acoustics, Speech, and Signal Processing, pp. 17-21, ISBN: 0-7803-8484-9, Quebec, Canada, May 2004, Montreal, Institute of Electrical and Electronics Engineers, Piscataway

Shin, B.S. \& Shin, Y.G. (1998) Fast 3D Solid Model Reconstruction from Orthographic Views, Computer-Aided Design, Vol. 30, No. 1, (Jan. 1998) pp. 63-76, ISSN: 0010-4485

Shum, H. \& Komura, T. (2004) A Spatiotemporal Approach to Extract the 3D Trajectory of the Baseball from a Single View Video Sequence, Proceedings of the IEEE International Conference on Multimedia and Expo, pp. 1583-1586, ISBN: 0-7803-8603-5, Taipei, Taiwan, June 2004, Institute of Electrical and Electronics Engineers, Piscataway

Theobalt, C.; Albrecht, I.; Haber, J.; Magnor, M. \& Seidel, H.P. (2004) Pitching a Baseball: Tracking High-Speed Motion with Multi-Exposure Images, ACM Transactions on Graphics, Vol. 23, No. 3, (Aug. 2004) pp. 540-547, ISSN: 0730-0301

Wang, C.K. ; Cheng, M.Y.; Liao, C.H.; Li, C.C.; Sun, C.Y. \& Tsai, M.C. (2004) Design and Implementation of a Multi-Purpose Real-Time Pan-Tilt Visual Tracking System, Proceedings of the IEEE International Conference on Control Applications, pp. 1079-1084, ISBN: 0-7803-8633-7, Taipei, Taiwan, Sep. 2004, Institute of Electrical and Electronics Engineers, Piscataw ay

Yan, X.; Yu, X. \& Hay, T.S. (2004) A 3D Reconstruction and Enrichment System for Broadcast Soccer Video, Proceedings of the 12th ACM International Conference on Multimedia, pp. 746-747, ISBN: 1-58113-893-8, New York, NY, USA, Oct. 2004, Institute of Electrical and Electronics Engineers, Piscataw ay

Yu, X.; Xu, C.; Tian, Q. \& Leong, H.W. (2003a) A Ball Tracking Framework for Broadcast Soccer Video, Proceedings of the IEEE International Conference on Multimedia and Expo, pp. 273-276, ISBN: 0-7803-7965-9, Baltimore, MD, USA, July 2003, Institute of Electrical and Electronics Engineers, Piscataw ay

Yu, X.; Leong, H.W.; Lim, J.; Tian, Q. \& Jiang, Z. (2003b) Team Possession Analysis for Broadcast Soccer Video Based on Ball Trajectory, Proceedings of the IEEE International Conference on Information, Communications and Signal Processing, pp. 1811-1815, ISBN: 0-7803-8185-8, Singapore, Dec. 2003, Institute of Electrical and Electronics Engineers, Piscataw ay 
Yu, X.; Sim, C.H.; Wang, J.R. \& Cheong, L.F. (2004) A Trajectory-Based Ball Detection and Tracking Algorithm in Broadcast Tennis Video, Proceedings of the IEEE International Conference on Image Processing, pp. 1049-1052, ISBN: 0-7803-8554-3, Genova, Italy, Oct. 2004, Institute of Electrical and Electronics Engineers, Piscataw ay 


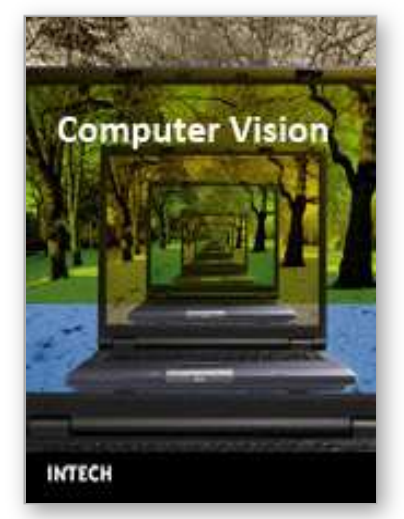

\author{
Computer Vision \\ Edited by Xiong Zhihui
}

ISBN 978-953-7619-21-3

Hard cover, 538 pages

Publisher InTech

Published online 01, November, 2008

Published in print edition November, 2008

This book presents research trends on computer vision, especially on application of robotics, and on advanced approachs for computer vision (such as omnidirectional vision). Among them, research on RFID technology integrating stereo vision to localize an indoor mobile robot is included in this book. Besides, this book includes many research on omnidirectional vision, and the combination of omnidirectional vision with robotics. This book features representative work on the computer vision, and it puts more focus on robotics vision and omnidirectioal vision. The intended audience is anyone who wishes to become familiar with the latest research work on computer vision, especially its applications on robots. The contents of this book allow the reader to know more technical aspects and applications of computer vision. Researchers and instructors will benefit from this book.

\title{
How to reference
}

In order to correctly reference this scholarly work, feel free to copy and paste the following:

Feng-Li Lian and Shih-Yuan Peng (2008). Image Acquisition Rate Control Based on Object State Information in Physical and Image Coordinates, Computer Vision, Xiong Zhihui (Ed.), ISBN: 978-953-7619-21-3, InTech, Available from:

http://www.intechopen.com/books/computer_vision/image_acquisition_rate_control_based_on_object_state_in formation_in_physical_and_image_coordinates

\section{INTECH}

open science | open minds

\section{InTech Europe}

University Campus STeP Ri

Slavka Krautzeka 83/A

51000 Rijeka, Croatia

Phone: +385 (51) 770447

Fax: +385 (51) 686166

www.intechopen.com

\section{InTech China}

Unit 405, Office Block, Hotel Equatorial Shanghai No.65, Yan An Road (West), Shanghai, 200040, China 中国上海市延安西路65号上海国际贵都大饭店办公楼405单元 Phone: +86-21-62489820

Fax: $+86-21-62489821$ 
(C) 2008 The Author(s). Licensee IntechOpen. This chapter is distributed under the terms of the Creative Commons Attribution-NonCommercialShareAlike-3.0 License, which permits use, distribution and reproduction for non-commercial purposes, provided the original is properly cited and derivative works building on this content are distributed under the same license. 